\title{
The Accessory Helix of Complexin Stabilizes a Partially Unzippered State of the SNARE Complex and Mediates the Complexin Clamping Function In Vivo
}

\author{
Joshua Brady, ${ }^{1}$ Alexander Vasin, ${ }^{2}$ and Maria Bykhovskaia ${ }^{1,2}$
}

https://doi.org/10.1523/ENEURO.0526-20.2021

${ }^{1}$ Ophthalmology, Visual and Anatomical Sciences Department, Wayne State University School of Medicine, Detroit, MI 48202 and ${ }^{2}$ Neurology Department, Wayne State University School of Medicine, Detroit, MI 48202

\begin{abstract}
Spontaneous synaptic transmission is regulated by the protein complexin (Cpx). Cpx binds the SNARE complex, a coil-coiled four-helical bundle that mediates the attachment of a synaptic vesicle (SV) to the presynaptic membrane (PM). Cpx is thought to clamp spontaneous fusion events by stabilizing a partially unraveled state of the SNARE bundle; however, the molecular detail of this mechanism is still debated. We combined electrophysiology, molecular modeling, and site-directed mutagenesis in Drosophila to develop and validate the atomic model of the Cpx-mediated clamped state of the SNARE complex. We took advantage of botulinum neurotoxins (BoNTs) B and $\mathrm{G}$, which cleave the SNARE protein synaptobrevin (Syb) at different sites. Monitoring synaptic depression on BoNT loading revealed that the clamped state of the SNARE complex has two or three unraveled helical turns of Syb. Sitedirected mutagenesis showed that the Cpx clamping function is predominantly maintained by its accessory helix $(\mathrm{AH})$, while molecular modeling suggested that the $\mathrm{Cpx} \mathrm{AH}$ interacts with the unraveled $\mathrm{C}$ terminus of Syb and the SV lipid bilayer. The developed molecular model was employed to design new Cpx poor-clamp and super-clamp mutations and to tested the predictions in silico employing molecular dynamics simulations. Subsequently, we generated Drosophila lines harboring these mutations and confirmed the poor-clamp and super-clamp phenotypes in vivo. Altogether, these results validate the atomic model of the $\mathrm{Cpx}$-mediated fusion clamp, wherein the $\mathrm{Cpx} \mathrm{AH}$ inserts between the SNARE bundle and the SV lipid bilayer, simultaneously binding the unraveled C terminus of Syb and preventing full SNARE assembly.
\end{abstract}

Key words: botulinum toxin; lipid bilayer; mEPSP; molecular dynamics; spontaneous transmission; synaptic vesicle

\section{Significance Statement}

Spontaneous release of neuronal transmitters is an important component of synaptic transmission, which controls neuronal development, homeostasis, and synaptic plasticity. The synaptic protein complexin (Cpx) regulates synaptic transmission by clamping spontaneous release. We developed and validated an atomic model of the Cpx-mediated clamping mechanism, which can be employed as a tool for generating predictions for site-directed mutagenesis and selectively manipulating spontaneous synaptic transmission.

\section{Introduction}

Neuronal transmitters are packed in synaptic vesicles (SVs) and released by fusion of SVs with the presynaptic membrane (PM). The attachment of an SV to the PM is

Received December 3, 2020; accepted February 9, 2021; First published March 9, 2021.

The authors declare no competing financial interests. mediated by the SNARE complex (Südhof and Rothman, 2009; Südhof, 2013), a coil-coiled four-stranded helical bundle, which consists of an SV protein synaptobrevin (Syb) or v-SNARE, and PM-associated proteins, syntaxin

Author contributions: A.V. and M.B. designed research; J.B., A.V., and M.B. performed research; J.B. and M.B. analyzed data; J.B. and M.B. wrote the paper. 
(Syx) and SNAP25 or t-SNARE. The assembly of the SNARE bundle enables overcoming the electrostatic and hydration repulsion between the SV and PM lipid bilayers (Rizo and Xu, 2015). The rapid synchronous fusion of SVs with the PM is evoked by an influx of $\mathrm{Ca}^{2+}$ ions into the nerve terminal, and a protein Synaptotagmin 1 acts as a $\mathrm{Ca}^{2+}$ sensor (Chapman, 2008).

Fusion events can also occur in the absence of action potentials, in a spontaneous mode. Genetic, biochemical, and physiological studies have identified a cytosolic protein complexin (Cpx) as a molecular player that inhibits spontaneous fusion but promotes the evoked $\mathrm{Ca}^{2+}$-dependent release (Mohrmann et al., 2015; Trimbuch and Rosenmund, 2016). Cpx attaches to the SNARE complex forming a five-helical bundle (Chen et al., 2002). Cpx deletion produces a drastic increase in spontaneous fusion events (Huntwork and Littleton, 2007), suggesting that the energetic barrier for SV fusion is reduced (Giraudo et al., 2006). This function of Cpx as a fusion clamp is well established in invertebrates (Hobson et al., 2011; Martin et al., 2011; Jorquera et al., 2012; Wragg et al., 2013), but is more controversial in mammalian synapses (Trimbuch and Rosenmund, 2016). However, it was demonstrated that Cpx inhibits spontaneous activity at cortical neuronal cultures (Yang et al., 2013), at mammalian calyx of Held (Chang et al., 2015), and at ribbon synapses (Vaithianathan et al., 2013, 2015). Furthermore, promoting Cpx action by either genetic overexpression or supplementation inhibited exocytosis in neurosecretory cells (Itakura et al., 1999; Abderrahmani et al., 2004; Liu et al., 2007). Altogether a large body of literature shows that Cpx clamps spontaneous fusion both in vitro and in vivo (Mohrmann et al., 2015), although this Cpx function in vivo appears to be synapse specific and is more prominent in invertebrates.

Several important features of the clamping mechanism have been established: (1) different domains of Cpx control evoked and spontaneous transmission, and these two Cpx functions are not correlated (Xue et al., 2007; Cho et al., 2014); (2) Cpx clamping function is regulated by its accessory helix (AH; Kaeser-Woo et al., 2012; Cho et al., 2014; Vasin et al., 2016) and by its C-terminal domain (Kaeser-Woo et al., 2012); (3) lipid binding is important for the Cpx clamping function (Wragg et al., 2013); (4) single point mutations in the $\mathrm{Cpx}$ AH selectively alter the spontaneous release component (Cho et al., 2014; Vasin et al., 2016); and (5) replacing the $\mathrm{Cpx} \mathrm{AH}$ by a non-native helix restores the clamping function (Radoff et al., 2014). Notably, the latter two findings appear to contradict each

This work was supported by National Science Foundation Grant IOS 1145010. Acknowledgements: MD simulations were performed at the Anton2 supercomputer (D.E. Shaw Research and Pittsburgh Supercomputer Center) and at XSEDE resources (Stampede supercomputer at TACC).

Correspondence should be addressed to Maria Bykhovskaia at mbykhovs@ med.wayne.edu

https://doi.org/10.1523/ENEURO.0526-20.2021

Copyright @ 2021 Brady et al.

This is an open-access article distributed under the terms of the Creative Commons Attribution 4.0 International license, which permits unrestricted use, distribution and reproduction in any medium provided that the original work is properly attributed. other, raising the question of the mechanism by which the $\mathrm{Cpx} \mathrm{AH}$ controls spontaneous transmission.

Although multiple studies suggest that $\mathrm{Cpx}$ interferes with zippering of v-SNARE onto t-SNARE (Giraudo et al., 2006, 2009; Krishnakumar et al., 2011, 2015; Kümmel et al., 2011; Li et al., 2011; Bykhovskaia et al., 2013; Vasin et al., 2016; Zdanowicz et al., 2017), the exact structure of the partially unraveled SNARE bundle in its clamped state is still debated. It was initially proposed that v-SNARE is unraveled radically, up to the middle of the four-helical bundle (Giraudo et al., 2009; Krishnakumar et al., 2011; Kümmel et al., 2011; Li et al., 2011). However, it was also shown that the electrostatic repulsion between the PM and an SV decays rapidly with distancing (Bykhovskaia et al., 2013; Fortoul et al., 2015) and therefore it is unlikely to drive the separation between the bilayers required for such radical SNARE unzippering. Other studies (Bykhovskaia et al., 2013; Fortoul et al., 2015, 2018; Vasin et al., 2016) suggested that a more likely scenario is that only two or three membrane-proximal helical turns (layers 7-9) of v-SNARE are separated from t-SNARE. Botulinum neurotoxins (BoNTs) represent an advantageous tool to discriminate between these different scenarios, since BoNTs cleave the SNARE proteins at distinct peptide bonds, and the cleavage depends on the BoNT serotype (Montecucco and Schiavo, 1993; Link et al., 1994). Here, we combined BoNT loading, molecular modeling of proteinlipid complexes, and $\mathrm{Cpx}$ mutagenesis to build and validate a model of the Cpx-mediated fusion clamp.

\section{Materials and Methods}

\section{Drosophila stocks and genetics}

All Drosophila melanogaster fly stocks were cultured on standard medium at a temperature of $22^{\circ} \mathrm{C}$. The following stocks were used: $c p x$ null mutant $c p x-/-\left[c p x^{S H 1}\right.$; Huntwork and Littleton (2007); obtained from the lab of J. T. Littleton]; wild-type (WT) Canton-S and C155 elev-Gal4 (Bloomington Drosophila Stock Center, Indiana University). Epoch Life Science was used for site directed mutagenesis of $\mathrm{Cpx}$ (Isoform Cpx 7A; Buhl et al., 2013). The PCR products were subcloned into a pValum construct, which enabled the use of the Gal4/UAS system for gene expression (Brand and Perrimon, 1993). The constructs were injected by BestGene for a targeted third chromosome insertion into yv;;attp2 site. Homozygote third chromosome UAS lines were recombined into the $c p x-/-$ null background ( $c p x^{S H 1}$; Huntwork and Littleton, 2007). The C155 elav-Gal4 driver was used to panneuronally express the mutated transgenes.

\section{Electrophysiology}

The third instar larvae were dissected in HL3 solution composed of the following: $70 \mathrm{~mm} \mathrm{NaCl}, 5 \mathrm{~mm} \mathrm{KCl}, 20 \mathrm{~mm}$ $\mathrm{MgCl}_{2}, 10 \mathrm{~mm} \mathrm{NaHCO}$, $5 \mathrm{~mm}$ trehalose, $115 \mathrm{~mm}$ sucrose, $2.5 \mathrm{~mm}$ HEPES- $\mathrm{HCl}, 2.5 \mathrm{~mm}$ HEPES- $\mathrm{NaOH}$, and $1 \mathrm{~mm}$ $\mathrm{CaCl}_{2}$ (pH 7.2-7.4). EPSPs and miniature EPSPs (mEPSPs) were recorded from neuromuscular junctions (NMJs) using the focal macropatch technique (Vasin and Bykhovskaia, 2017). To record selectively from $1 \mathrm{~b}$ type boutons, we used DIC optics and 60x water immersion objective (Olympus 
0.95 NA) with a 2-mm working distance. Recordings of excitatory postsynaptic potentials (EPSPs) and miniature EPSPs (mEPSPs) were performed with macropatch electrodes with tip diameters of $5 \mu \mathrm{m}$ and 1-M $\Omega$ seal resistances and digitized using a Digitdata A/D board and Axoscope software (Molecular Devices). Nerve stimulation was performed with Master-8 pulse stimulator (AMPI). The recordings were analyzed using Quantan software (Bykhovskaia, 2008).

\section{Loading nerve terminals with BoNTs}

BoNT serotypes BoNT/B or BoNT/G (1 mg/ml, Metabiologics Inc) mixed (1:1) with fluorescent dye rhodamine $B$ isothiocyanate (Sigma-Aldrich) conjugated to a 10-kDa dextran (RITC-dextran, concentration between 40 and $200 \mu \mathrm{m}$ ) were loaded into nerve terminals through the cut axon. We have adopted the protocol developed for loading $\mathrm{Ca}^{2+}$ indicators in Drosophila terminals (Rossano and Macleod, 2007). The second abdominal muscle segment was used in these experiments to minimize the potential variability. The axon was cut to a length of $\sim 0.5 \mathrm{~mm}$ from the site of the muscle innervation and inserted into the suction electrode. The suction electrodes were fire polished and had the opening diameter of 2-3 $\mu \mathrm{m}$, so that the axon would fit tightly and there would be no solution exchange between the bath and the suction electrode. The suction electrode was filled with the mixture of BoNT and RITC, positioned near the cut axon, and the axon was rapidly suctioned into the electrode. Subsequently, the recording electrode was rapidly positioned over a $1 \mathrm{~b}$ bouton as close to the suction electrode as possible to minimize the BoNT diffusion time Evoked transmission was elicited via the same suction electrode.

\section{Immunohistochemistry}

Dissected larvae were fixed for $45 \mathrm{~min}$ in HL3 saline containing $4 \%$ formaldehyde. Following washing in PBST ( $0.1 \%$ Triton $\mathrm{X}-100$ containing $1 \times$ PBS solution), larvae were preincubated in the blocking solution containing $2 \%$ normal goat serum, $2 \%$ bovine serum albumin, and $0.05 \%$ sodium azide for $1 \mathrm{~h}$. Primary antibody was applied overnight at $4^{\circ} \mathrm{C}$. The secondary antibody was applied for 4-6 $\mathrm{h}$ at the room temperature.

For Cpx immunostaining, Drosophila anti-Cpx antibody (1:200), a generous gift from J. T. Littleton (Huntwork and Littleton, 2007; Cho et al., 2014) and a secondary goat anti-rabbit polyclonal Cy3 IgG antibody (1:200, Novus Biologicals) were used. For the horseradish peroxidase (HRP) labeling, the preparations were incubated with anti-HRP conjugated to Alexa Fluor 488 (1:250, Jackson ImmunoResearch). To detect BoNT, we used a polyclonal monovalent antibody specific to BoNT/B (1:100, Metabiologics) and a secondary mouse antirabbit IgG antibody (1:100, Life Technologies).

Fluorescence was visualized and imaged using Velocity software (Improvision) on a laser-based confocal microscope (PerkinElmer) with an ORCA-ER CCD camera (Hamamatsu) using an oil-immersion $50 \times / 0.9$ objective (Olympus).

\section{Molecular dynamics}

The molecular systems were constructed using Visual Molecular Dynamics Software (VMD, Theoretical and Computational Biophysics Group, NIH Center for Macromolecular Modeling and Bioinformatics, at the Beckman Institute, University of Illinois at UrbanaChampaign). All the simulations were performed in a water/ion environment with explicit waters. Potassium and chloride ions were added to neutralize the systems and to yield a $150 \mathrm{~mm}$ concentration of $\mathrm{KCl}$. Water boxes with added ions were constructed using VMD. The phosphatidylcholine (POPC) lipid bilayers mimicking an SV were generated using VMD. The initial structure of anionic lipid bilayer containing phosphatidylserine (POPS) and phosphatidylinositol 4,5-bisphosphate $\left(\mathrm{PIP}_{2}\right)$, POPC: POPS:PIP 2 (75:20:5) mimicking the PM (Alwarawrah and Wereszczynski, 2017) was kindly provided by J. Wereszczynski (Illinois Institute of Technology). In all the systems, the lipid bilayers were positioned in the $x y$-plane.

The MD simulations were performed employing CHARMM36 force field (Vanommeslaeghe et al., 2010) modified to include the parameters for $\mathrm{PIP}_{2}$ as described previously (Alwarawrah and Wereszczynski, 2017). The simulations were performed with periodic boundary conditions and Ewald electrostatics in the NPT ensemble at $310 \mathrm{~K}$. The heating (20 ps) and equilibration (100 ns) phases were performed employing NAMD (Phillips et al., 2005) Scalable Molecular Dynamics (Theoretical and Computational Biophysics Group, NIH Center for Macromolecular Modeling and Bioinformatics, at the Beckman Institute, University of Illinois at UrbanaChampaign) at XSEDE (Extreme Science and Engineering Discovery Environment) Stampede cluster (TACC). The NAMD simulations were performed with a flexible cell and with a time-step of $1.5 \mathrm{fs}$, employing Langevin thermostat and Berendsen barostat. Production runs were performed at Anton2 supercomputer (Shaw, 2014; Shaw et al., 2009) with Desmond software through the MMBioS (National Center for Multiscale Modeling of Biological Systems, Pittsburgh Supercomputing Center and D.E. Shaw Research Institute). All the Anton2 simulations were performed in a semi-isotropic regime, with a time-step of $2.5 \mathrm{fs}$, and employing the multigrator (Lippert et al., 2013) to maintain constant temperature and pressure. The trajectory analysis was performed employing VMD and Vega ZZ (Drug Design Laboratory) software. All the parameters along all the trajectories were computed with a time step of $2.4 \mathrm{~ns}$.

\section{Statistical analysis}

One-way ANOVA followed by the Tukey's test was employed to evaluate statistical significance.

\section{Results}

We took advantage of botulinum toxins BoNT/B (Schiavo et al., 1992) and BoNT/G (Yamasaki et al., 1994), which cleave Syb at two different sites (Fig. 1A). Transgenic expression of BoNTs in Drosophila demonstrated that both 
A

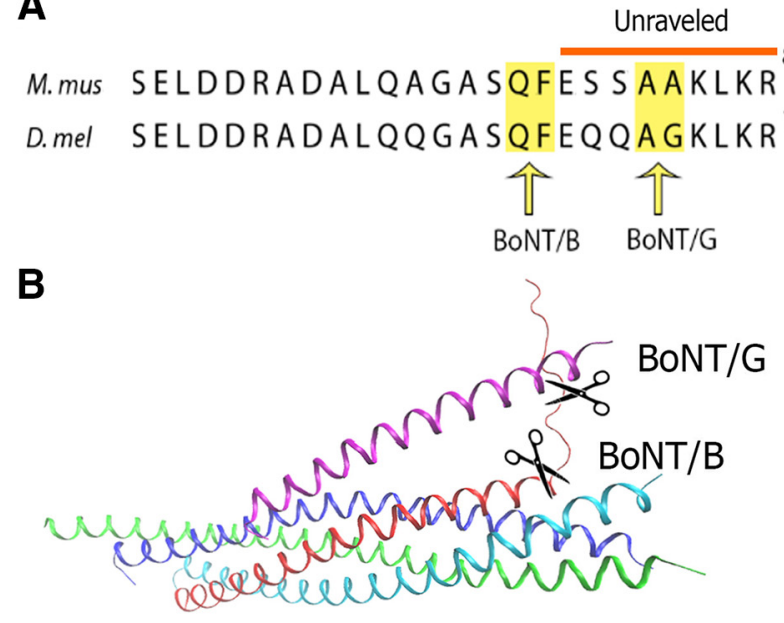

C

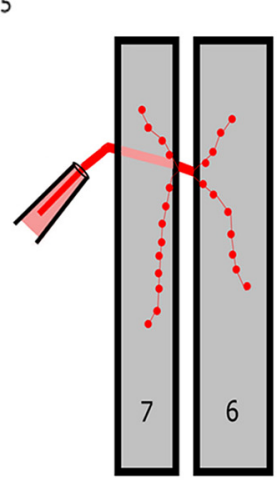

2

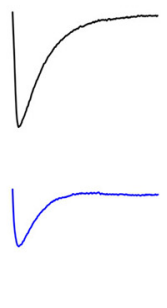

5
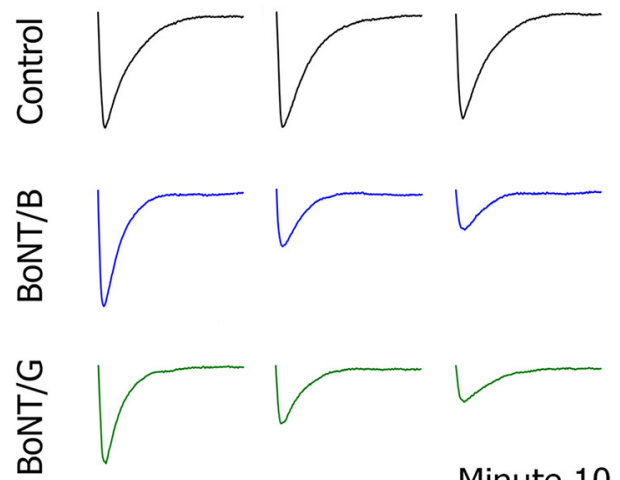

Minute 10

H

G

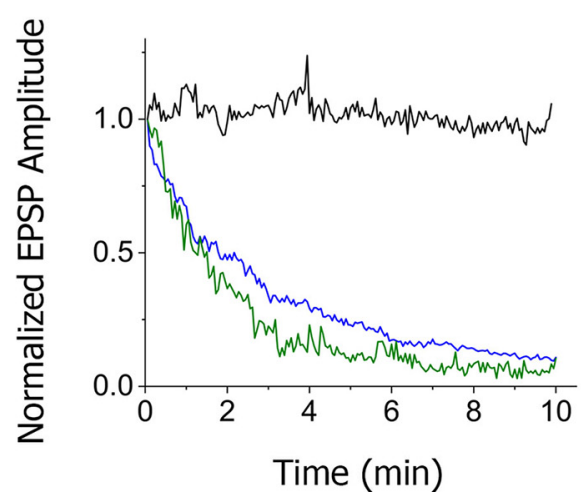

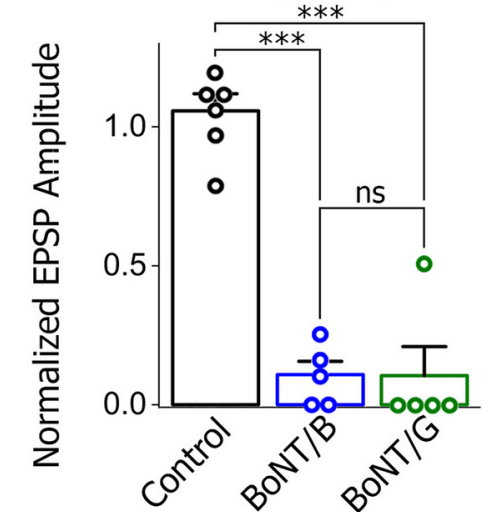

Figure 1. Loading BoNT in the nerve terminals. A, Cleavage sites for BoNT/B and BoNT/G in the mammalian Syb and Drosophila $\mathrm{n}$-syb. B. The sites of BoNT/B and BoNT/G cleavage shown at the model of the clamped state of the SNARE-Cpx complex. Note that the BoNT/G cleavage site is situated within the unraveled C terminus of Syb, while the BoNT/B cleavage site is situated within the zippered Syb region. $\boldsymbol{C}$, The diagram illustrating the protocol of the BoNT loading through the cut axon innervating the muscles 6 and 7 of the Drosophila NMJ. $\boldsymbol{D}$, The RITC dye mixed with BoNT diffused through the entire NMJ on BoNT loading. $\boldsymbol{E}$, Immunostaining shows the presence of BoNT/B in the nerve terminals in the end of the experiment. $\boldsymbol{F}-\boldsymbol{H}$, Evoked transmission is rapidly reduced on BoNT loading. Examples of recorded EPSPs $(\boldsymbol{F})$ illustrate that EPSP amplitude diminishes within minutes in BoNT/B and BoNT/G loaded preparations (blue and green), but not in control baseline recordings (black). The kinetics of the EPSP decay (G) shows a rapid decrease for both BoNT/B (blue) and BoNT/G (green) versus control. The data are normalized by the EPSP amplitude of the first response. Each data point represents an average of five experiments. In the end of the recording $(\boldsymbol{H})$ the EPSP amplitude in BoNT loaded preparations is significantly reduced compared with the baseline control; ${ }^{\star \star \star} p<0.001$. ns - not significant. 
serotypes cleave the Drosophila neuronal Syb (n-syb; Backhaus et al., 2016). Our initial goal was to test the "late clamp" model (Bykhovskaia et al., 2013; Vasin et al., 2016), which proposed that the clamped state of the SNARE-Cpx complex involves the unraveled layers 7-9 of the SNARE bundle (Fig. 1A,B). As such, this model predicts that n-syb will be accessible for the cleavage by BoNT/G but not by BoNT/B (Fig. 1B) when the SNARE complex is clamped. Conversely, a more radically unzippered n-syb would be accessible to cleavage by BoNT/B.

BoNT was loaded through the cut axon (Fig. 1C; Rossano and Macleod, 2007). To monitor loading, we added RITC to the solution and ensured that the dye reached the nerve terminals (Fig. 1D). To ensure the presence of BoNT in the terminals, at the end of the recordings we fixed the preparations and performed immunostaining for BoNT/B (Fig. 1E). In addition, we assessed BoNT activity by monitoring evoked release on the nerve stimulation, since at these conditions the pool of docked SVs is rapidly depleted, and therefore the EPSP amplitude should decay rapidly (Hua et al., 1998). To assess whether this is the case, we stimulated the nerve continuously at a 3- $\mathrm{Hz}$ frequency while loading either BoNT/B or BoNT/G. For both BoNT serotypes, we observed rapid decay in EPSP amplitudes (Fig. 1F), and within $10 \mathrm{~min}$ the evoked activity was almost entirely eliminated (Fig. 1F,G).

Next, we loaded the preparations with BoNT/B or BoNT/G and monitored the decay in spontaneous synaptic activity. BoNT/G loading produced a rapid decay in spontaneous transmission (Fig. $2 A-C$, green). In contrast, there was essentially no decay after BoNT/B loading within 20 min (Fig. $2 A-C$, blue). These results suggest that $n-$ syb is accessible for BoNT/G cleavage but to a lesser extent for BoNT/B cleavage. This is consistent with the model wherein BoNT/B does not cleave the clamped SNARE complexes of docked SVs (Fig. 2D, blue), while BoNT/G does (Fig. 2D, green). Indeed, the rate of spontaneous transmission is low $(\sim 0.7-0.8 \mathrm{~Hz}$ per bouton; Vasin et al., 2016) and the number of SVs docked to the PM is large ( 1000 per bouton; Meinertzhagen et al., 1998; Sabeva et al., 2017), and therefore docked SVs can maintain spontaneous transmission for 20 min or longer, accounting for steady spontaneous transmission in BoNT/B loaded preparations.

Since the model proposes that the clamped state of the SNARE complex with partially unraveled Syb is stabilized by $\mathrm{Cpx}$, it could be expected that the differential effects of BoNT/G and BoNT/B serotypes would be abolished for $c p x-/-$ NMJs. To test whether this is the case, we recorded spontaneous transmission from $c p x-/-$ NMJs on BoNT/G versus BoNT/B loading. We found that the differential effect of the BoNT/B and BoNT/G serotypes was completely abolished in $c p x-/-$ preparations (Fig. 2E-G). Indeed, spontaneous transmission at $c p x-/-$ NMJs decayed rapidly on loading either BoNT/G or BoNT/B. This could be expected because of high rates of spontaneous transmission in cpx-/ - NMJs ( $~ 80 \mathrm{~Hz}$; Vasin et al., 2016), which should rapidly deplete the pool of docked SVs.

We next employed Cpx mutagenesis to delineate the role of the $\mathrm{Cpx} \mathrm{AH}$ in the clamping mechanism. The following mutants have been generated (Fig. $3 A$ ): (1) deletion of the $\mathrm{Cpx} A \mathrm{H}$ and $\mathrm{N}$ terminus $\left(c p x^{\Delta(A H+N)}\right.$, residues $4-48)$; (2) deletion of the $\mathrm{N}$ terminus only $\left(c p x^{\Delta N}\right.$, residues 4$35)$; and (3) replacement of the $\mathrm{Cpx} A \mathrm{H}$ by the poly $\mathrm{A}$ sequence ( $c p x^{A H(p o l y ~ A)}$, residues $37-48$ being replaced). All the mutated Cpx forms were expressed in the $c p x-/-$ background. We have ascertained that the mutated proteins are properly expressed in the nerve terminals (Fig. 3B).

We found that the mEPSP frequency in the $c p x^{\Delta(A H+N)}$ mutant was $\sim 17$-fold higher than in the control rescue line (Fig. 3C-E, ochre vs orange), although it was not as high as in $c p x-/-(\sim 80 \mathrm{~Hz}$; Vasin et al., 2016). Interestingly, the $c p x^{\Delta N}$ mutant (Fig. 3C,D, pink) had an unaltered mEPSP frequency compared with the control, showing that the spontaneous activity was inhibited by the $\mathrm{Cpx} A \mathrm{H}$ region. Notably, replacing the native $A H$ sequence with the poly $A$ sequence in the $\left.c p x^{A H(p o l y ~} A\right)$ mutant decreased the mEPSP frequency significantly below the control level (Fig. 3C, $D$, maroon vs orange). None of the mutants showed any significant alterations in either mEPSP amplitude (Fig. 3E) or evoked transmission (Fig. 3F,G) compared with the rescue control.

These results clearly show that the $\mathrm{Cpx} A \mathrm{H}$ is required for the Cpx clamping function while the $\mathrm{Cpx} \mathrm{N}$-terminal domain has no role in it. Further, these results show that the structure of the $\mathrm{Cpx} \mathrm{AH}$ is fine-tuned for incomplete clamping, since its replacement with a poly $A$ sequence actually enhances the Cpx clamping function. How could the poly $A$ sequence be more functional than the native sequence? The simplest explanation is that the $\mathrm{Cpx} A \mathrm{H}$ is inserted between the SNARE bundle and an SV thus creating an extra barrier between the PM and an SV. Since the poly A sequence is hydrophobic, it would likely have a high affinity to an SV and could therefore create a more reliable barrier than the native $\mathrm{Cpx} \mathrm{AH}$. To explore this possibility, we performed molecular modeling of the SNARE-Cpx complex between lipid bilayers mimicking the PM and an SV.

The structure of the Drosophila SNARE-Cpx bundle with partially unraveled n-syb (Vasin et al., 2016) was positioned between the bilayers. The distance between the bilayers was adjusted to $3 \mathrm{~nm}$ since at this distance the bilayers do not interfere with the SNARE-Cpx bundle, the C-terminal residues of $n$-syb become embedded in the SV bilayer, and the electrostatic repulsion between the bilayers is not prominent (Bykhovskaia et al., 2013; Fortoul et al., 2015). We then performed 300 ns of MD simulations for this molecular system. The system remained stable, the $\mathrm{Cpx} \mathrm{AH}$ remained positioned between the SNARE bundle and the SV bilayer, the unraveled $C$ terminus of $n$-syb (layers 7-9) remained separated from the bundle, and the $\mathrm{C}$-terminal residues of $\mathrm{n}$-syb remained embedded in the SV bilayer (Fig. 4A). We then replaced the $\mathrm{Cpx} \mathrm{AH}$ (residues 37-48) with the poly $\mathrm{A}$ sequence and repeated the simulations. Notably, we found that at the end of the trajectory the mutated $\mathrm{Cpx} \mathrm{AH}$ interacted with the bilayer mimicking an SV more tightly, reinforcing the barrier between the bilayers (Fig. 4B, arrow).

We next asked whether this model of the fusion clamp (Fig. 4A) has predictive power and could guide us in the Cpx mutagenesis. To test this, we designed two point mutations in the $\mathrm{Cpx} \mathrm{AH}$ : poor-clamp and super-clamp. To 
A
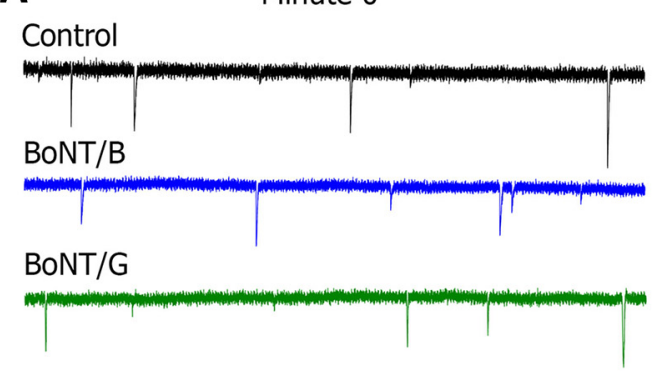

B

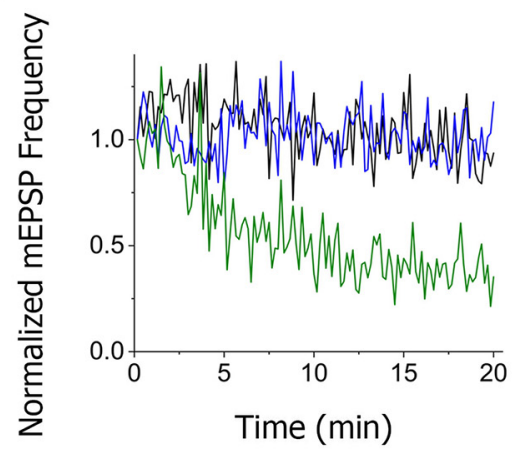

Minute 20
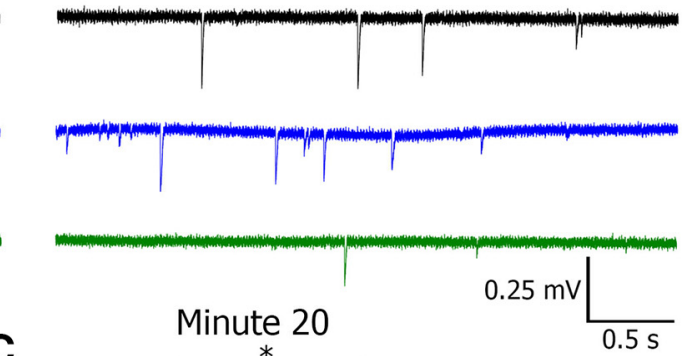

C

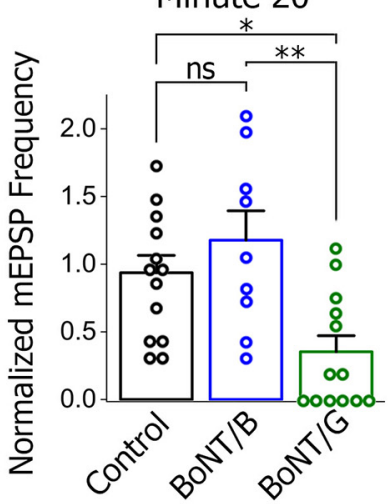

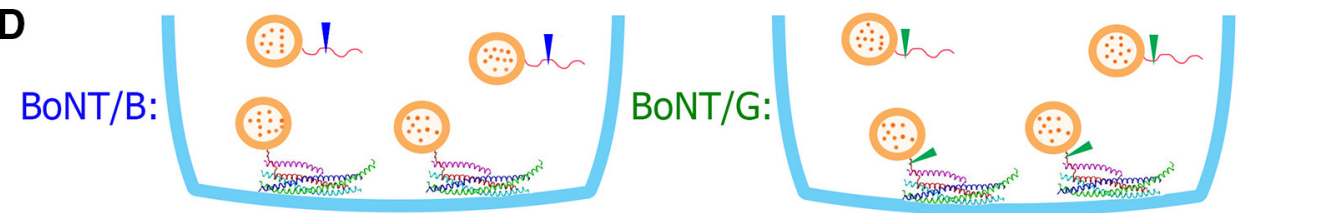

\section{E Minute 0 \\ Control

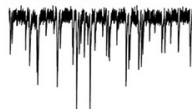

BoNT/B

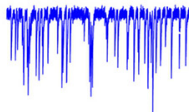

BoNT/G

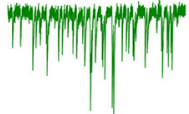

Minute 20
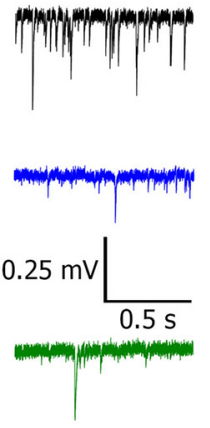

$\mathbf{F}$

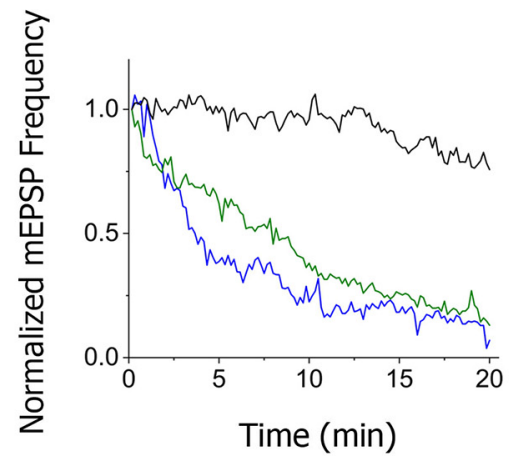

G

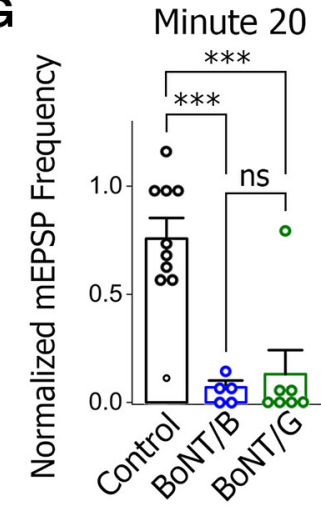

Figure 2. Spontaneous transmission decays on BoNT/G but not on BoNT/B loading, and the differential effect of BoNTs is abolished in cpx-/- NMJs. A-C, In WT preparations, spontaneous transmission significantly decays within 20 min BoNT/G but not BoNT/B loading. Representative traces $(\boldsymbol{A})$ that illustrate that after $20 \mathrm{~min}$ of BoNT loading spontaneous transmission is reduced in the BoNT/G loaded but not in the BoNT/B loaded NMJ. The mEPSP frequency is reduced over time (B) in BoNT/G loaded but not in BoNT/B loaded preparations. The mEPSP frequency is binned over 10-s intervals and normalized by the first bin in each experiment. In the end of 20-min loading, the mEPSP frequency is significantly reduced compared with the control baseline in BoNT/G but not in BoNT/B loaded preparations; ${ }^{*} p<0.05$, ${ }^{* *} p<0.01,{ }^{* \star *} p<0.001$. $\boldsymbol{D}$, The diagram illustrating that BoNT/G but not BoNT/B would cleave Syb of the clamped SNARE-Cpx complexes of docked SVs. $\boldsymbol{E}-\boldsymbol{G}$, The $c p x-/-$ preparations show rapid decay in the mEPSP frequency on BoNT loading because of high rates of spontaneous transmission. However, the differential effect of BoNT/B versus BoNT/G is abolished. ${ }^{\star \star \star} p<0.001$. Representative traces $(\boldsymbol{E})$ illustrate the reduction in spontaneous transmission after 20 min BoNT loading. The mEPSP frequency is rapidly reduced over time $(\boldsymbol{F})$ in BoNT/G and BoNT/B preparations. In the end of 20-min loading, the mEPSP frequency is significantly reduced compared with the control baseline in both BoNT/G and BoNT/B loaded preparations. ns - not significant. 
A
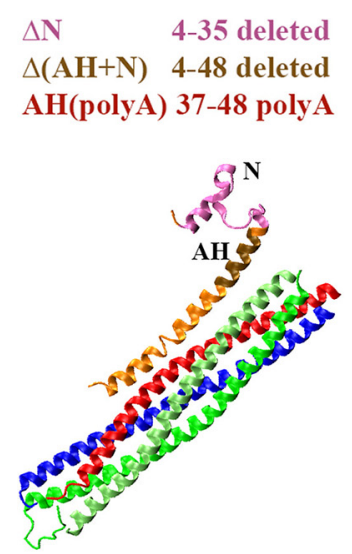

C
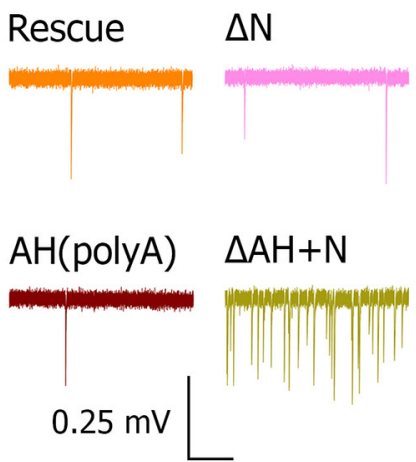

$0.5 \mathrm{~s}$

$\mathbf{F}$

Rescue
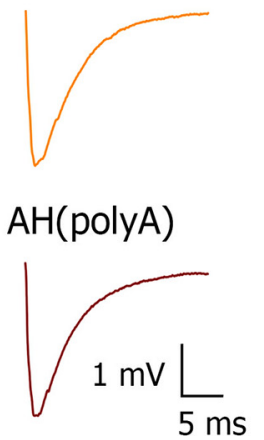

B

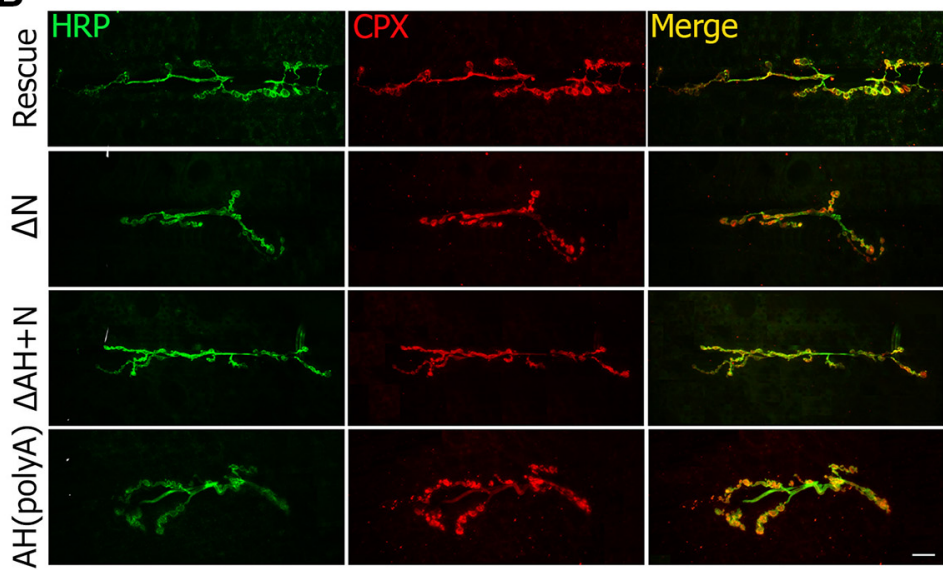

D

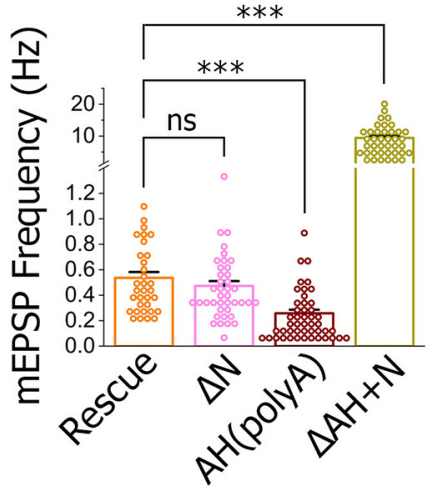

$E$

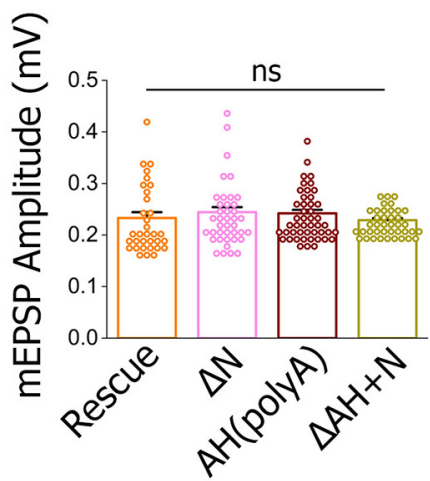

G

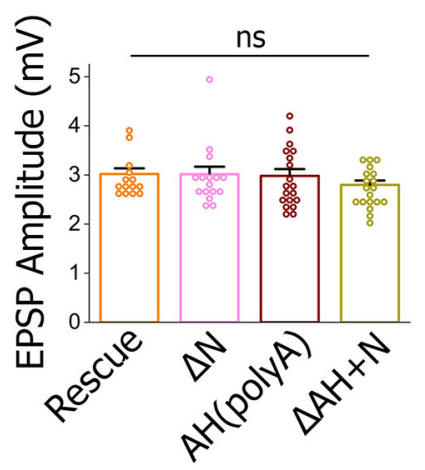

Figure 3. $\mathrm{Cpx} \mathrm{AH}$ has a pivotal role in the regulation of spontaneous transmission. $\boldsymbol{A}$, The diagram showing the design of the mutations in relevance to the structure of the SNARE-Cpx complex. Red: Syb; blue: Syx; green: SNAP25; orange: Cpx. B, All the mutants show a normal Cpx expression pattern, with Cpx localized to the nerve terminals. Images show the double labeling for the neuronal marker HRP and immunostaining for $\mathrm{Cpx}$, which are co-localized. Scale bar: $10 \mu \mathrm{m}$. $\boldsymbol{C}, \boldsymbol{D}, \mathrm{Cpx}$ AH but not N-terminal domain regulates the frequency of spontaneous transmission. Representative traces $(\boldsymbol{C})$ and mEPSP frequencies $(\boldsymbol{D})$ show that spontaneous transmission is not altered in the $c p x^{\Delta N}$ mutant (pink), is significantly reduced in the $\left.c p x^{A H(p o l y} A\right)$ mutant (maroon), and is drastically enhanced in the $c p x^{\Delta(N+A H)}$ mutant (ochre); ${ }^{\star \star \star} p<0.001$. $\boldsymbol{E}$, The mutations do not alter mEPSP amplitudes. $\boldsymbol{F}$, G, Evoked transmission is not altered in either of the mutants. Representative traces $(\boldsymbol{F})$ and EPSP amplitudes $(\boldsymbol{G})$ do not show any significant alterations in either of the mutants. ns - not significant.

design the poor-clamp mutation, we examined the atomic model of the clamped SNARE-Cpx complex to identify the $\mathrm{Cpx}$ residues which stabilize the unraveled state of $\mathrm{n}$ syb. We found that these interactions (Fig. 5A) predominantly involve the residues R43 and Q44 of the $\mathrm{Cpx} A H$
(RQ motif; Fig. $5 A$, boxed region), which are conserved between mammalian and Drosophila Cpx forms (R37 and Q38 in the mammalian Cpx1). Therefore, the model predicts that $c P x^{R Q->A A}$ mutation would produce the poorclamp phenotype. 

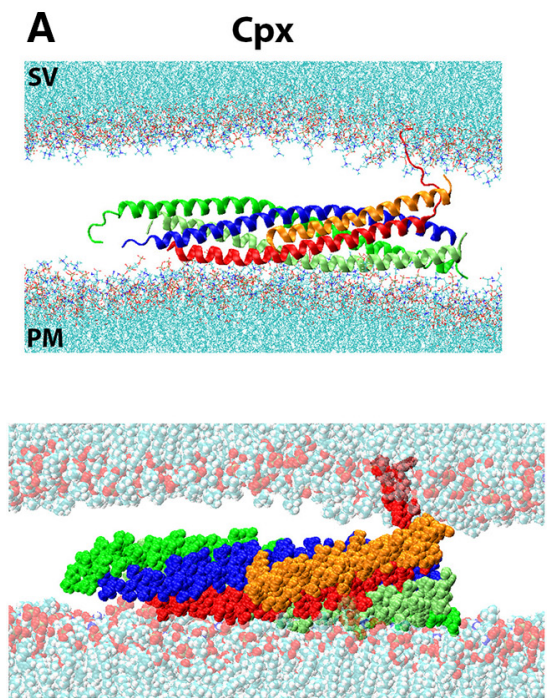
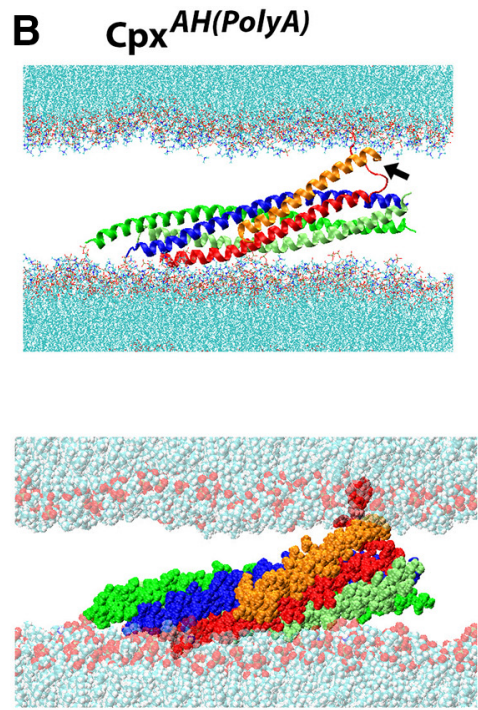

Figure 4. The all-atom model of the clamped SNARE-Cpx complex between lipid bilayers mimicking the PM and an SV. Cartoon (top) and VdW (bottom) representations of the SNARE-Cpx complex are shown. $\boldsymbol{A}$, Native Cpx (orange) stabilizes the unraveled C terminus of Syb (red) and also interacts with the SV bilayer. $\boldsymbol{B}$, The Cpx ${ }^{\mathrm{AH}(\text { poly } A)}$ mutant interacts tightly with the SV bilayer (arrow) enhancing the barrier between the SV and the PM.

The model also predicts that the super-clamp phenotype can be created by loosening the attachment of the $\mathrm{Cpx} \mathrm{AH}$ to the SNARE bundle. Such mutation would fortify the clamp by separating the $\mathrm{Cpx} \mathrm{AH}$ from the bundle, enhancing the interactions of the $\mathrm{Cpx} \mathrm{AH}$ with the SV lipid bilayer, and thus augmenting the barrier between the SNARE bundle and the SV, similar to the $c p x^{A H(p o l y ~ A)}$ mutant (Fig. 4B). The examination of the atomic model revealed tight links between the SNARE bundle and the IK motif of Cpx (Fig. 5B, residues 147 and K48, homologous to L41 and R42 in mammalian Cpx1). Therefore, it could be predicted that mutating this motif would enhance the clamping function of $\mathrm{Cpx}$.

First, we tested these predictions in silico. The molecular systems (Fig. 4A) with the mutated Cpx forms (Cpx ${ }^{R Q-}$ $>A A$, poor-clamp and $\mathrm{Cpx}{ }^{I K->A A}$, super-clamp) were generated, and prolonged MD simulations (for $1.2 \mu \mathrm{s}$ ) for each system were performed. To monitor the separation of $n$-syb from the SNARE bundle, we measured the distance between $\mathrm{C} \alpha$ atoms of the $\mathrm{C}$-terminal residues of $\mathrm{n}$ syb an Syx along each trajectory. In this in silico molecular system, zippering of $n$-syb onto t-SNARE would serve as a measure for the ability of the SNARE complex to fully assemble and drive the SV-PM fusion. We found that the molecular system with the Cpx $x^{I K->A A}$ mutant had n-syb separated from the t-SNARE bundle for the entire length of the trajectory (Fig. 5C, navy), and this clamped state was supported by the $\mathrm{Cpx} \mathrm{AH}$, which interacted with the unraveled $\mathrm{C}$ terminus of $\mathrm{n}$-syb and with the SV bilayer (Fig. 5D, top). In contrast, the system containing the $C p x^{R Q->A A}$ mutant did not stabilize. In this system, the unraveled $C$ terminus of $n$-syb separated from the bilayer mimicking an SV and attached to the SNARE bundle (Fig. $5 C$, magenta, $D$, bottom), although full SNARE zippering was not observed at our time-scale $(1.2 \mu \mathrm{s})$. Finally, the system containing native $\mathrm{Cpx}$ showed an intermediate behavior, with the $C$ terminus of $n$-syb remaining partially unraveled at the end of the trajectory (Fig. $5 C$, orange, $D$, middle). These results demonstrate in silico that the $\mathrm{Cpx}{ }^{\prime K->A A}$ mutant would likely stabilize the clamped state of the SNARE complex, producing the super-clamp phenotype, while the $C p x^{R Q->A A}$ mutant would not be able to maintain the clamp and would likely show the poor-clamp phenotype.

We then tested these predictions in vivo. The mutated $c p x$ genes $c p x^{I K->A A}$ and $c p x^{R Q-P A A}$ were expressed in the $c p x-/-$ background. Both mutated forms of $\mathrm{Cpx}$ were properly expressed in the nerve terminals (Fig. 6A). We then assayed spontaneous transmission in both lines. We found that the mEPSP frequency was more than doubled in the $c P x^{R Q->A A}$ mutant compared with the control rescue line (Fig. 6B,C). Thus, as predicted by the model, the $c p x^{R Q->A A}$ mutant showed the poor-clamp phenotype, although quantitatively the effect of the RQ$>\mathrm{AA}$ mutation in the $\mathrm{Cpx} \mathrm{AH}$ was approximately 8-fold below the effect of the $\mathrm{Cpx} \mathrm{AH}$ deletion (Fig. 3). In contrast, the mEPSP frequency was significantly reduced in the $c p x^{I K->A A}$ mutant showing, as predicted, the superclamp phenotype. Neither mEPSP amplitude (Fig. $6 D$ ) nor evoked transmission (Fig. 6E) was affected in either of the mutants.

Altogether, our results support a model of the clamped state of the SNARE complex with only two or three C-terminal layers of Syb being unraveled. Our results also suggest that this clamped state of the SNARE complex is stabilized by the Cpx AH being inserted between the SNARE bundle and the SV lipid bilayer, while also interacting with the partially unraveled $C$ terminus of Syb. Finally, we show that this molecular model enables designing poor-clamp and super-clamp mutations and thus selectively manipulating Cpx clamping function and spontaneous synaptic transmission. 
A n-syb-Cpx interations supporting the clamp

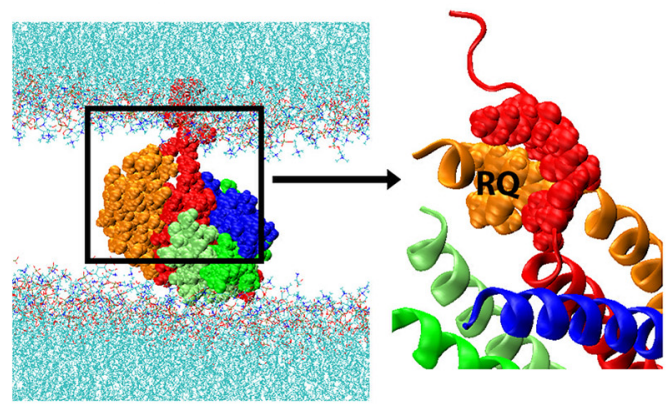

C

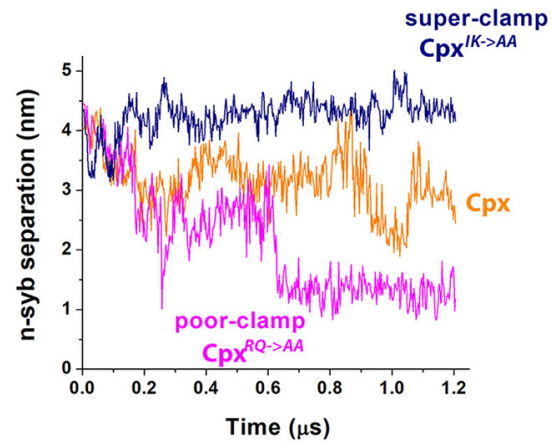

\section{B n-syb-Cpx interations counteracting the clamp}

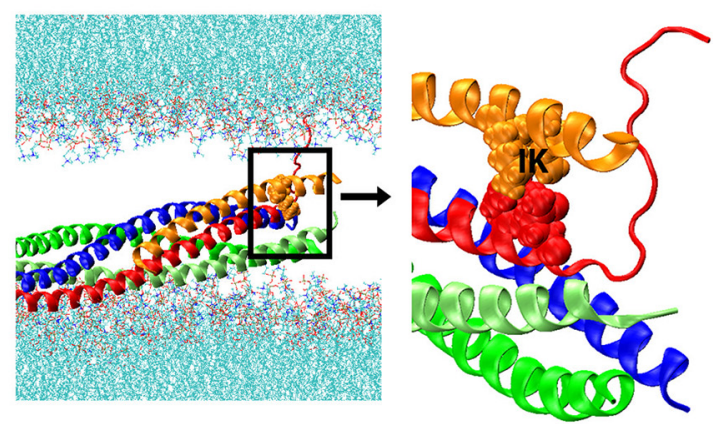

D

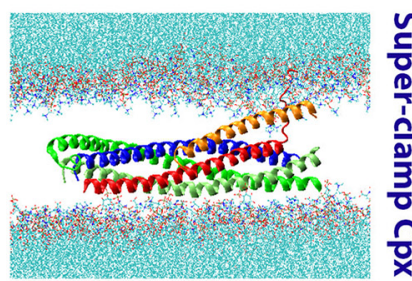

n
$\frac{0}{0}$
$\frac{1}{1}$
$\frac{n}{2}$
$\frac{3}{0}$
$\frac{n}{0}$
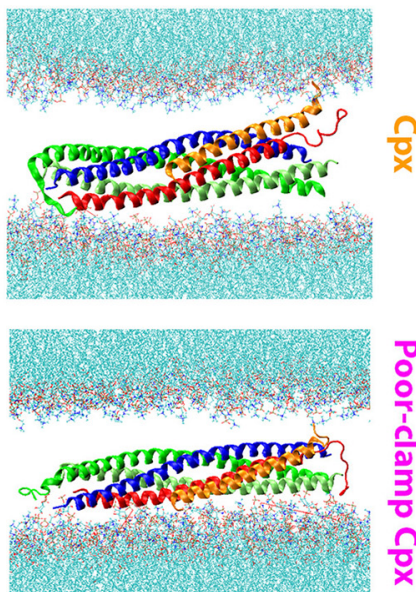

Figure 5. The design and in silico testing of the Cpx poor-clamp and super-clamp mutants. $\boldsymbol{A}$, The poor-clamp mutant is designed to disrupt the $R Q$ motif within the $\mathrm{Cpx} A H$, which maintains the interactions between the $\mathrm{Cpx} A H$ and the unraveled n-syb terminus. $\boldsymbol{B}$, The super-clamp mutant is designed to disrupt the IK motif maintaining the interactions between the Cpx AH and the zippered part of the SNARE bundle. C, MD simulations of the native and mutated complexes show that the super-clamp mutant maintains the separation between the unraveled $n$-syb $\mathrm{C}$ terminus and the SNARE bundle, while the poor-clamp mutant does not. The graph shows the distance between $\mathrm{C}_{\alpha}$ atoms of the C-terminal residues of $\mathrm{n}$-syb and Syx over the length of the 1.2- $\mu \mathrm{s}$ MD trajectory. Trajectory points are separated by 240 ps. $\boldsymbol{D}$. Structures of the mutated and native Cpx complexes in the end of respective MD trajectories. Note the unraveled $\mathrm{C}$ terminus of $\mathrm{n}$-syb and the $\mathrm{Cpx} \mathrm{AH}$ tightly interacting with the SV bilayer in the super-clamp mutant. In contrast, in the poor-clamp mutant, the $\mathrm{C}$ terminus of $\mathrm{n}$-syb separated from the SV bilayer and started forming contacts with tSNARE.

\section{Discussion}

Cpx is a potent regulator of synaptic transmission, and its function has been studied extensively (Mohrmann et al., 2015; Trimbuch and Rosenmund, 2016). Several molecular models have been developed to address the mechanism by which $\mathrm{Cpx}$ inhibits spontaneous transmission (Giraudo et al., 2009; Kümmel et al., 2011; Li et al., 2011; Bykhovskaia et al., 2013). Most of the models agree that Cpx stabilizes a partially unraveled state of the SNARE complex, thus preventing full SNARE zippering and spontaneous fusion events. However, the specific molecular detail of this mechanism is still debated.
Further, it is largely agreed that the $\mathrm{Cpx} \mathrm{AH}$ has a pivotal role in the clamping mechanism; however, it is not yet clear how specifically it mediates the Cpx clumping function.

One of the earliest models (Giraudo et al., 2009) suggested that the $\mathrm{Cpx} \mathrm{AH}$ intercalates between the unraveled Syb and the t-SNARE bundle. Subsequently, this model was modified to incorporate several SNARE complexes cross-linked by $\mathrm{Cpx}$ molecules (Kümmel et al., 2011; Krishnakumar et al., 2015). A more recent study proposed that Cpx competes with Syb for binding to tSNARE and thus inhibits SNARE assembly (Zdanowicz et 
A

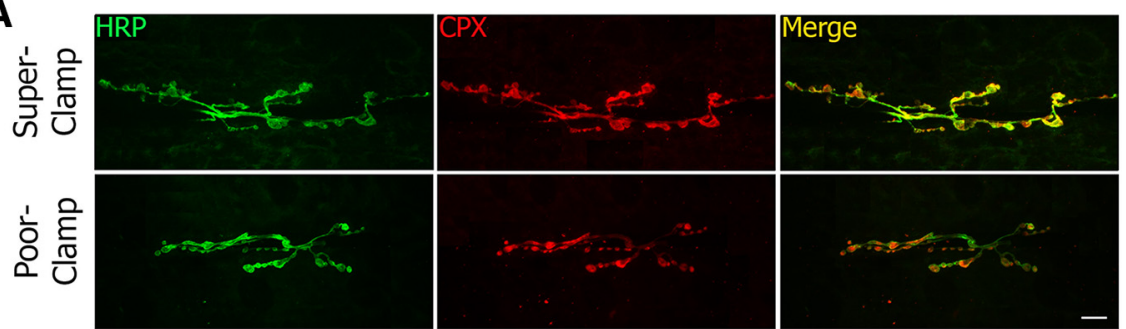

B
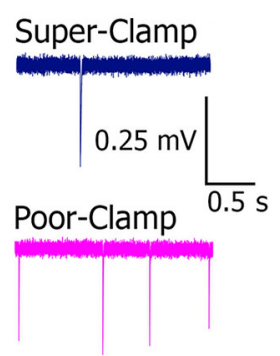

E
C

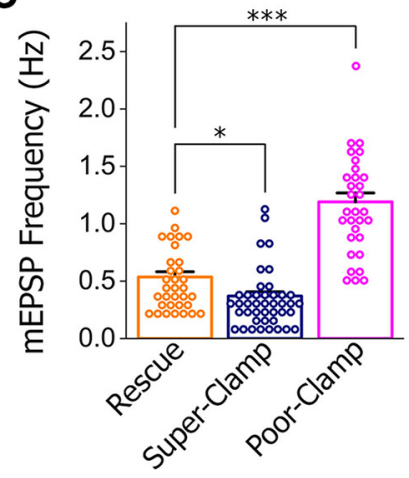

D

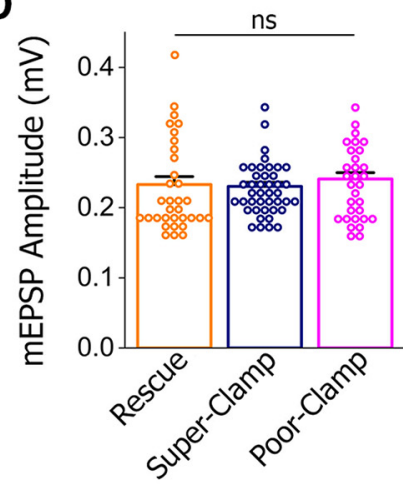

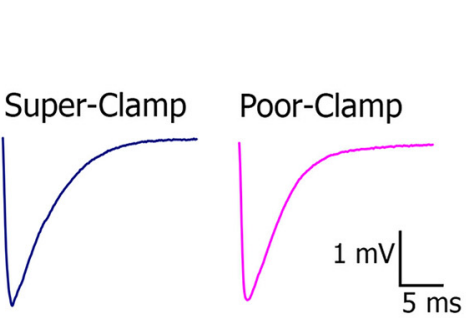

$\mathbf{F}$

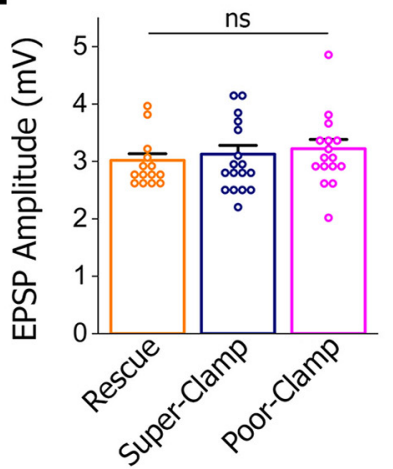

Figure 6. Testing the super-clamp and poor-clamp mutations in vivo. $\boldsymbol{A}$, Both mutants show a normal Cpx expression pattern, with Cpx co-localized with the neuronal marker HRP. Scale bar: $10 \mu \mathrm{m}$. $\boldsymbol{B}, \boldsymbol{C}$, Recording traces $(\boldsymbol{B})$ and EPSP frequencies $(\boldsymbol{C})$ show that spontaneous transmission is significantly increased in the poor-clamp mutant and significantly decreased in the super-clamp mutant; ${ }^{\star} p<0.05,{ }^{* \star *} p<0.001$. $\boldsymbol{D}$, mEPSP amplitudes are not affected by the mutations. $\boldsymbol{E}, \boldsymbol{F}$, The mutations do not affect evoked transmission, as evident from representative EPSP traces $(\boldsymbol{E})$ and average EPSP amplitudes $(\boldsymbol{F})$. ns - not significant.

al., 2017). The studies cited above suggested that Cpx radically interferes with the SNARE assembly, preventing the major part of Syb from zippering onto the t-SNARE bundle. An alternative late clamp model (Bykhovskaia et al., 2013; Vasin et al., 2016) proposed that Cpx only interferes with the final stages of SNARE zippering, inhibiting two or three SV-proximal helical turns of Syb from zippering onto t-SNARE.

Interestingly, an in vivo study in Caenorhabditis elegans demonstrated that the replacement of the $\mathrm{Cpx} \mathrm{AH}$ with a non-native helix (multiple repeats of the EAAK motif) did not change spontaneous transmission (Radoff et al., 2014). This study suggested that it is the helical structure of the $\mathrm{Cpx} \mathrm{AH}$ and not its specific sequence which is critical for the Cpx clamping function. However, this finding appears to be in contradiction with several in vivo studies in mouse (Yang et al., 2010) and Drosophila (Cho et al., 2014; Vasin et al., 2016) which showed that point mutations within the $\mathrm{Cpx} \mathrm{AH}$ sequence can produce poor-clamp or super-clam phenotypes.

We took advantage of the Drosophila preparation, which shows a very strong and robust effect of $\mathrm{Cpx}$ on spontaneous transmission (Huntwork and Littleton, 2007; Jorquera et al., 2012). The first question we asked was: how radically is the SNARE complex unzippered in its clamped state? To elucidate this question, we took advantage of clostridial neurotoxins (Niemann et al., 1994; Pellegrini et al., 1995; Xu et al., 1998; Hua and Charlton, 1999), a tool which has been broadly used to understand how SNARE proteins control exocytosis. We used BoNT serotypes B and G, which cleave Syb ( $\mathrm{n}$-syb in Drosophila) at two different sites: near layer 7 (BoNT/G) and near layer 6 (BoNT/B). We reasoned that if Syb is unzippered by only two or three helical turns in its clamped state, as suggested by the late clamp model (Bykhovskaia et al., 2013), then we would observe a faster depression of spontaneous release 
on BoNT/G loading since BoNT/G (but not BoNT/B) would cleave docked SVs attached to the PM by partially unraveled SNARE complexes. In contrast, if Syb is separated from t-SNARE more radically, as suggested by other models (Giraudo et al., 2009; Kümmel et al., 2011), then both BoNT serotypes would cleave partially zippered SNARE complexes and, respectively, produce similar decays in spontaneous transmission. Our results supported the first scenario, with BoNT/G producing a significantly stronger reduction in spontaneous transmission than BoNT/B.

In line with these findings, our $\mathrm{Cpx}$ mutagenesis experiments coupled with molecular modeling showed that the region of the $\mathrm{Cpx} \mathrm{AH}$ (11 residues forming three helical turns) is critical for the Cpx clamping function, and that this $\mathrm{Cpx}$ region would likely interact with the unraveled region of Syb. Indeed, removal of the $\mathrm{Cpx} \mathrm{AH}$ produced an $\sim 17$-fold increase in the mEPSP frequency. In should be noted, however, that this increase in spontaneous synaptic activity did not approach in magnitude the effect of the Cpx deletion (Huntwork and Littleton, 2007; Jorquera et al., 2012), which showed over 100 increase in the mEPSP frequency when measured by the same technique as in the present study (Vasin et al., 2016). Thus, although Cpx AH represents an important structural element for clamping spontaneous fusion, it does not completely account for this $\mathrm{Cpx}$ action. What other Cpx motifs may contribute to fusion clamping? Our study showed that this Cpx function does not depend on its $\mathrm{N}$-terminal domain, since its deletion did not alter spontaneous transmission. However, an earlier study at the mouse cultured neurons demonstrated that the Cpx clamping function depends on its C-terminal domain (Kaeser-Woo et al., 2012). Further investigation is needed to understand how the $\mathrm{Cpx} \mathrm{AH}$ and its $\mathrm{C}$-terminal domain interact in controlling spontaneous transmission.

Notably, we also found that replacing this $\mathrm{Cpx}$ region with a poly $A$ sequence significantly reduces the mEPSP frequency compared with the control rescue line. This finding agrees with the results obtained in C. elegans, which showed that replacing the $\mathrm{Cpx} \mathrm{AH}$ with a non-native helix rescues the Cpx function (Radoff et al., 2014). Furthermore, our finding shows that a non-native helix, such as the poly $A$ sequence, can clamp spontaneous transmission even better than the native $\mathrm{Cpx} \mathrm{AH}$. This result suggests that the $\mathrm{Cpx}$ sequence is fine-tuned to regulate the rate of spontaneous transmission but not designed to maximally block it. This is not surprising, since the spontaneous release is an important component of synaptic transmission, required for neuronal development and several forms of plasticity (Kavalali, 2015; Andreae and Burrone, 2018).

To understand how the $\mathrm{Cpx} \mathrm{AH}$ clamps fusion, we have developed a molecular model of the SNARE-Cpx complex between lipid bilayers mimicking an SV and the PM. Our modeling suggested that the $\mathrm{Cpx} \mathrm{AH}$ could clamp fusion by creating a barrier between the SNARE bundle and the SV bilayer while simultaneously interacting with the unraveled $\mathrm{C}$ terminus of Syb. These three-partied interactions, involving the partially unraveled $C$ terminus of Syb, the $\mathrm{Cpx} \mathrm{AH}$, and the SV bilayer would stabilize the clamped state of the SNARE complex. In this model, a more hydrophobic $\mathrm{Cpx} \mathrm{AH}$ (such as the poly A sequence) could produce a better clamp because of more tight interactions with the SV bilayer.

We then combined computations and experiments to test whether this model could guide us in targeted mutagenesis. To produce the poor-clamp phenotype, we mutated two residues within the $\mathrm{Cpx} A \mathrm{H}\left(\mathrm{Cpx}^{R Q->A A}\right)$ which were predicted to bind the unraveled Syb terminus. Testing this mutation in silico suggested that indeed, the poor- clamp mutation would accelerate SNARE zippering. We next generated the Drosophila line harboring the poor-clamp mutation and demonstrated that the spontaneous transmission in this mutant was increased more than twice compared with the control rescue. One could argue that the mutation could reduce the $\mathrm{Cpx}$ expression levels, however, this explanation is highly unlikely. Indeed, earlier studies (Cho et al., 2014; Vasin et al., 2016) showed that mutations within the $\mathrm{Cpx} \mathrm{AH}$ do not affect either $\mathrm{Cpx}$ expression or delivery to the nerve terminals, and the immunolabeling data obtained in the present study argues that this not the case for the poor-clamp $\mathrm{Cpx}{ }^{R Q->A A} \mathrm{mu}$ tant. Instead, our results suggest that the poor-clamp phenotype is produced by the compromised interactions of the $\mathrm{Cpx} \mathrm{AH}$ with the n-syb unraveled terminus, as predicted by the model. Notably, the quantitative effect of the poor-clamp mutation ( $\sim 2$-fold increase) is much smaller than the effect of the $\mathrm{Cpx}$ AH deletion ( $\sim 17$-fold). This result is in line with our model, which suggests that the clamping mechanism enabled by the $\mathrm{Cpx} \mathrm{AH}$ includes two components: (1) binding the partially unraveled $\mathrm{C}$ terminus of n-syb and preventing its full zippering; and (2) creating a barrier between the SV and the SNARE bundle and thus separating the SV from the PM. These two mechanisms are likely to act synergistically. Indeed, the attachment of the $\mathrm{Cpx} \mathrm{AH}$ to an SV would promote the separation of the Cpx AH from the SNARE bundle and, respectively would stabilize the partially unzippered state of Syb. Since the $C p x^{R Q->A A}$ mutant can only compromise the first but not the second mechanism, it is not surprising that the effect produced by this mutation is modest. The atomic model developed here enables systematic targeted manipulations by the two mechanisms and evaluating their quantitative impact. For example, the model predicts that the fusion clamp can be enhanced by fortifying the interactions of the $\mathrm{Cpx} \mathrm{AH}$ with the SV bilayer by substituting the $\mathrm{Cpx}$ residues that face away from the SNARE bundle by more hydrophobic residues, such as Ala, Phe, Trp, or Leu. Furthermore, the model also predicts that the fusion clamp can be fortified by weakening the interactions between $\mathrm{Cpx} \mathrm{AH}$ and the SNARE bundle.

To test the latter prediction, we designed the superclamp mutation by disrupting the interactions of the $\mathrm{Cpx}$ $\mathrm{AH}$ with the zippered part of the SNARE bundle, thus promoting the interactions of the $\mathrm{Cpx} A \mathrm{H}$ which mediate the fusion clamp. A similar strategy for designing superclamp single point mutations in $\mathrm{Cpx}$ and $\mathrm{n}$-syb was employed in an earlier study (Vasin et al., 2016). Testing the super-clamp mutation in silico showed that the mutation would slow down zippering of the SNARE complex, and the Drosophila line harboring the super-clamp mutation 
showed a significant and selective decrease in the spontaneous transmission.

These results promote mechanistic understanding of how $\mathrm{Cpx}$ interactions fine-tune spontaneous synaptic transmission and provide the strategy for selectively manipulating the spontaneous release component.

\section{References}

Abderrahmani A, Niederhauser G, Plaisance V, Roehrich M-E, Lenain V, Coppola T, Regazzi R, Waeber G (2004) Complexin I regulates glucose-induced secretion in pancreatic beta-cells. J Cell Sci 117:2239-2247.

Alwarawrah M, Wereszczynski J (2017) Investigation of the effect of bilayer composition on $\mathrm{PKC} \alpha-\mathrm{C} 2$ domain docking using molecular dynamics simulations. J Phys Chem B 121:78-88.

Andreae LC, Burrone J (2018) The role of spontaneous neurotransmission in synapse and circuit development. J Neurosci Res 96:354-359.

Backhaus P, Langenhan T, Neuser K (2016) Effects of transgenic expression of botulinum toxins in Drosophila. J Neurogenet 30:2231.

Brand AH, Perrimon N (1993) Targeted gene expression as a means of altering cell fates and generating dominant phenotypes. Development 118:401-415.

Buhl LK, Jorquera RA, Akbergenova Y, Huntwork-Rodriguez S, Volfson D, Littleton JT (2013) Differential regulation of evoked and spontaneous neurotransmitter release by C-terminal modifications of complexin. Mol Cell Neurosci 52:161-172.

Bykhovskaia M (2008) Making quantal analysis more convenient, fast, and accurate: user-friendly software QUANTAN. J Neurosci Methods 168:500-513.

Bykhovskaia M, Jagota A, Gonzalez A, Vasin A, Littleton JT (2013) Interaction of the complexin accessory helix with the C-terminus of the SNARE complex: molecular-dynamics model of the fusion clamp. Biophys J 105:679-690.

Chang S, Reim K, Pedersen M, Neher E, Brose N, Taschenberger H (2015) Complexin stabilizes newly primed synaptic vesicles and prevents their premature fusion at the mouse calyx of held synapse. J Neurosci 35:8272-8290.

Chapman ER (2008) How does synaptotagmin trigger neurotransmitter release? Annu Rev Biochem 77:615-641.

Chen X, Tomchick DR, Kovrigin E, Araç D, Machius M, Südhof TC, Rizo J (2002) Three-dimensional structure of the complexin/ SNARE complex. Neuron 33:397-409.

Cho RW, Kümmel D, Li F, Baguley SW, Coleman J, Rothman JE, Littleton JT (2014) Genetic analysis of the Complexin trans-clamping model for cross-linking SNARE complexes in vivo. Proc Natl Acad Sci USA 111:10317-10322.

Fortoul N, Singh P, Hui CY, Bykhovskaia M, Jagota A (2015) Coarsegrained model of SNARE-mediated docking. Biophys J 108:22582269.

Fortoul N, Bykhovskaia M, Jagota A (2018) Coarse-grained model for zippering of SNARE from partially assembled states. J Phys Chem B 122:10834-10840.

Giraudo CG, Eng WS, Melia TJ, Rothman JE (2006) A clamping mechanism involved in SNARE-dependent exocytosis. Science 313:676-680.

Giraudo CG, Garcia-Diaz A, Eng WS, Chen Y, Hendrickson WA, Melia TJ, Rothman JE (2009) Alternative zippering as an on-off switch for SNARE-mediated fusion. Science 323:512-516.

Hobson RJ, Liu Q, Watanabe S, Jorgensen EM (2011) Complexin maintains vesicles in the primed state in $C$. elegans. Curr Biol 21:106-113.

Hua SY, Charlton MP (1999) Activity-dependent changes in partial VAMP complexes during neurotransmitter release. Nat Neurosci 2:1078-1083.
Hua SY, Raciborska DA, Trimble WS, Charlton MP (1998) Different VAMP/synaptobrevin complexes for spontaneous and evoked transmitter release at the crayfish neuromuscular junction. J Neurophysiol 80:3233-3246.

Huntwork S, Littleton JT (2007) A complexin fusion clamp regulates spontaneous neurotransmitter release and synaptic growth. Nat Neurosci 10:1235-1237.

Itakura M, Misawa H, Sekiguchi M, Takahashi S, Takahashi M (1999) Transfection analysis of functional roles of complexin I and II in the exocytosis of two different types of secretory vesicles. Biochem Biophys Res Commun 265:691-696.

Jorquera RA, Huntwork-Rodriguez S, Akbergenova Y, Cho RW, Littleton JT (2012) Complexin controls spontaneous and evoked neurotransmitter release by regulating the timing and properties of synaptotagmin activity. J Neurosci 32:18234-18245.

Kaeser-Woo YJ, Yang X, Südhof TC (2012) C-terminal complexin sequence is selectively required for clamping and priming but not for $\mathrm{Ca} 2+$ triggering of synaptic exocytosis. J Neurosci 32:2877-2885.

Kavalali ET (2015) The mechanisms and functions of spontaneous neurotransmitter release. Nat Rev Neurosci 16:5-16.

Krishnakumar SS, Radoff DT, Kümmel D, Giraudo CG, Li F, Khandan L, Baguley SW, Coleman J, Reinisch KM, Pincet F, Rothman JE (2011) A conformational switch in complexin is required for synaptotagmin to trigger synaptic fusion. Nat Struct Mol Biol 18:934940.

Krishnakumar SS, Li F, Coleman J, Schauder CM, Kümmel D, Pincet F, Rothman JE, Reinisch KM (2015) Re-visiting the trans insertion model for complexin clamping. Elife 4:e04463.

Kümmel D, Krishnakumar SS, Radoff DT, Li F, Giraudo CG, Pincet F, Rothman JE, Reinisch KM (2011) Complexin cross-links prefusion SNAREs into a zigzag array. Nat Struct Mol Biol 18:927-933.

Li F, Pincet F, Perez E, Giraudo CG, Tareste D, Rothman JE (2011) Complexin activates and clamps SNAREpins by a common mechanism involving an intermediate energetic state. Nat Struct Mol Biol 18:941-946.

Link E, Blasi J, Chapman ER, Edelmann L, Baumeister A, Binz T, Yamasaki S, Niemann H, Jahn R (1994) Tetanus and botulinal neurotoxins. Tools to understand exocytosis in neurons. Adv Second Messenger Phosphoprotein Res 29:47-58.

Lippert RA, Predescu C, lerardi DJ, Mackenzie KM, Eastwood MP, Dror RO, Shaw DE (2013) Accurate and efficient integration for molecular dynamics simulations at constant temperature and pressure. J Chem Phys 139:164106.

Liu J, Guo T, Wu J, Bai X, Zhou Q, Sui SF (2007) Overexpression of complexin in PC12 cells inhibits exocytosis by preventing SNARE complex recycling. Biochemistry (Mosc) 72:439-444.

Martin JA, Hu Z, Fenz KM, Fernandez J, Dittman JS (2011) Complexin has opposite effects on two modes of synaptic vesicle fusion. Curr Biol 21:97-105.

Meinertzhagen IA, Govind CK, Stewart BA, Carter JM, Atwood HL (1998) Regulated spacing of synapses and presynaptic active zones at larval neuromuscular junctions in different genotypes of the flies Drosophila and Sarcophaga. J Comp Neurol 393:482492.

Mohrmann R, Dhara M, Bruns D (2015) Complexins: small but capable. Cell Mol Life Sci 72:4221-4235.

Montecucco C, Schiavo G (1993) Tetanus and botulism neurotoxins: a new group of zinc proteases. Trends Biochem Sci 18:324-327.

Niemann H, Blasi J, Jahn R (1994) Clostridial neurotoxins: new tools for dissecting exocytosis. Trends Cell Biol 4:179-185.

Pellegrini LL, O'Connor V, Lottspeich F, Betz H (1995) Clostridial neurotoxins compromise the stability of a low energy SNARE complex mediating NSF activation of synaptic vesicle fusion. EMBO J 14:4705-4713.

Phillips JC, Braun R, Wang W, Gumbart J, Tajkhorshid E, Villa E, Chipot C, Skeel RD, Kalé L, Schulten K (2005) Scalable molecular dynamics with NAMD. J Comput Chem 26:1781-1802.

Radoff DT, Dong Y, Snead D, Bai J, Eliezer D, Dittman JS (2014) The accessory helix of complexin functions by stabilizing central helix secondary structure. Elife 3:e04553. 
Rizo J, Xu J (2015) The synaptic vesicle release machinery. Annu Rev Biophys 44:339-367.

Rossano AJ, Macleod GT (2007) Loading Drosophila nerve terminals with calcium indicators. J Vis Exp. Advance online publication. Retrieved July 30, 2007. doi: 10.3791/250.

Sabeva N, Cho RW, Vasin A, Gonzalez A, Littleton JT, Bykhovskaia M (2017) Complexin mutants reveal partial segregation between recycling pathways that drive evoked and spontaneous neurotransmission. J Neurosci 37:383-396.

Schiavo G, Benfenati F, Poulain B, Rossetto O, Polverino de Laureto P, DasGupta BR, Montecucco C (1992) Tetanus and botulinum-B neurotoxins block neurotransmitter release by proteolytic cleavage of synaptobrevin. Nature 359:832-835.

Shaw DE (2014) Long molecular dynamics simulations of proteins: progress, promise, and problems. Abstr Pap Am Chem S 247.

Shaw DE, Dror RO, Salmon JK,Grossman JP, Mackenzie KM, Bank JA, Young C, Deneroff MM, Batson B, Bowers KJ, Chow E, Eastwood MP, lerardi DJ, Klepeis JL, Kuskin JS, Larson RH, Lindorff-Larsen K, Maragakis P, Moraes MA, Piana S, et al. (2009) Millisecond-scale molecular dynamics simulations on Anton. In: Proceedings of the Conference on High Performance Computing, Networking, Storage and Analysis. New York: ACM.

Südhof TC (2013) Neurotransmitter release: the last millisecond in the life of a synaptic vesicle. Neuron 80:675-690.

Südhof TC, Rothman JE (2009) Membrane fusion: grappling with SNARE and SM proteins. Science 323:474-477.

Trimbuch T, Rosenmund C (2016) Should I stop or should I go? The role of complexin in neurotransmitter release. Nat Rev Neurosci 17:118-125.

Vaithianathan T, Zanazzi G, Henry D, Akmentin W, Matthews G (2013) Stabilization of spontaneous neurotransmitter release at ribbon synapses by ribbon-specific subtypes of complexin. J Neurosci 33:8216-8226.

Vaithianathan T, Henry D, Akmentin W, Matthews G (2015) Functional roles of complexin in neurotransmitter release at ribbon synapses of mouse retinal bipolar neurons. J Neurosci 35:40654070
Vanommeslaeghe K, Hatcher E, Acharya C, Kundu S, Zhong S, Shim J, Darian E, Guvench O, Lopes P, Vorobyov I, Mackerell AD (2010) CHARMM general force field: a force field for drug-like molecules compatible with the CHARMM all-atom additive biological force fields. J Comput Chem 31:671-690.

Vasin A, Bykhovskaia M (2017) Focal macropatch recordings of synaptic currents from the Drosophila larval neuromuscular junction. J Vis Exp. Advance online publication. Retrieved September 25, 2017. doi: 10.3791/56493.

Vasin A, Volfson D, Littleton JT, Bykhovskaia M (2016) Interaction of the complexin accessory helix with synaptobrevin regulates spontaneous fusion. Biophys J 111:1954-1964.

Wragg RT, Snead D, Dong Y, Ramlall TF, Menon I, Bai J, Eliezer D, Dittman JS (2013) Synaptic vesicles position complexin to block spontaneous fusion. Neuron 77:323-334.

Xu T, Binz T, Niemann H, Neher E (1998) Multiple kinetic components of exocytosis distinguished by neurotoxin sensitivity. Nat Neurosci 1:192-200.

Xue M, Reim K, Chen X, Chao HT, Deng H, Rizo J, Brose N, Rosenmund C (2007) Distinct domains of complexin I differentially regulate neurotransmitter release. Nat Struct Mol Biol 14:949-958.

Yamasaki S, Binz T, Hayashi T, Szabo E, Yamasaki N, Eklund M, Jahn R, Niemann H (1994) Botulinum neurotoxin type G proteolyses the Ala81-Ala82 bond of rat synaptobrevin 2. Biochem Biophys Res Commun 200:829-835.

Yang X, Kaeser-Woo YJ, Pang ZP, Xu W, Südhof TC (2010) Complexin clamps asynchronous release by blocking a secondary $\mathrm{Ca}(2+)$ sensor via its accessory $\alpha$ helix. Neuron 68:907-920.

Yang X, Cao P, Südhof TC (2013) Deconstructing complexin function in activating and clamping $\mathrm{Ca} 2$ +-triggered exocytosis by comparing knockout and knockdown phenotypes. Proc Natl Acad Sci USA 110:20777-20782.

Zdanowicz R, Kreutzberger A, Liang B, Kiessling V, Tamm LK, Cafiso DS (2017) Complexin binding to membranes and acceptor tSNAREs explains its clamping effect on fusion. Biophys $\mathrm{J}$ 113:1235-1250. 